Article

\title{
The Italian Domination Numbers of Some Products of Directed Cycles
}

\section{Kijung Kim $\mathbb{D}$}

Department of Mathematics, Pusan National University, Busan 46241, Korea; knukkj@pusan.ac.kr

Received: 29 July 2020; Accepted: 31 August 2020; Published: 1 September 2020

\begin{abstract}
An Italian dominating function on a digraph $D$ with vertex set $V(D)$ is defined as a function $f: V(D) \rightarrow\{0,1,2\}$ such that every vertex $v \in V(D)$ with $f(v)=0$ has at least two in-neighbors assigned 1 under $f$ or one in-neighbor $w$ with $f(w)=2$. In this article, we determine the exact values of the Italian domination numbers of some products of directed cycles.
\end{abstract}

Keywords: Italian dominating function; Italian domination number; cartesian product; strong product MSC: 05C69

\section{Introduction and Preliminaries}

Let $D=(V, A)$ be a finite simple digraph with vertex set $V=V(D)$ and arc set $A=A(D)$. An arc joining $v$ to $w$ is denoted by $v \rightarrow w$. The maximum out-degree and maximum in-degree of $D$ are denoted by $\Delta^{+}(D)$ and $\Delta^{-}(D)$, respectively.

Let $D_{1}=\left(V_{1}, A_{1}\right)$ and $D_{2}=\left(V_{2}, A_{2}\right)$ be two digraphs. The cartesian product of $D_{1}$ and $D_{2}$ is the digraph $D_{1} \square D_{2}$ with vertex set $V_{1} \times V_{2}$ and for two vertices $\left(u_{1}, u_{2}\right)$ and $\left(v_{1}, v_{2}\right)$,

$$
\left(u_{1}, u_{2}\right) \rightarrow\left(v_{1}, v_{2}\right)
$$

if one of the following holds: (i) $u_{1}=v_{1}$ and $u_{2} \rightarrow v_{2}$; (ii) $u_{1} \rightarrow v_{1}$ and $u_{2}=v_{2}$.

The strong product of $D_{1}$ and $D_{2}$ is the digraph $D_{1} \otimes D_{2}$ with vertex set $V_{1} \times V_{2}$ and for two vertices $\left(u_{1}, u_{2}\right)$ and $\left(v_{1}, v_{2}\right)$,

$$
\left(u_{1}, u_{2}\right) \rightarrow\left(v_{1}, v_{2}\right)
$$

if one of the following holds: (i) $u_{1} \rightarrow v_{1}$ and $u_{2} \rightarrow v_{2}$; (ii) $u_{1}=v_{1}$ and $u_{2} \rightarrow v_{2}$; (iii) $u_{1} \rightarrow v_{1}$ and $u_{2}=v_{2}$.

An Italian dominating function (IDF) on a digraph $D$ is defined as a function $f: V(D) \rightarrow\{0,1,2\}$ such that every vertex $v \in V(D)$ with $f(v)=0$ has at least two in-neighbors assigned 1 under $f$ or one in-neighbor $w$ with $f(w)=2$. In other words, we say that a vertex $v$ for which $f(v) \in\{1,2\}$ dominates itself, while a vertex $v$ with $f(v)=0$ is dominated by $f$ if it has at least two in-neighbors assigned 1 under $f$ or one in-neighbor $w$ with $f(w)=2$. An Italian dominating function $f: V(D) \rightarrow\{0,1,2\}$ gives a partition $\left\{V_{0}, V_{1}, V_{2}\right\}$ of $V(D)$, where $V_{i}:=\{x \in V(D) \mid f(x)=i\}$. The weight of an Italian dominating function $f$ is the value $\omega(f)=f(V(D))=\sum_{u \in V(D)} f(u)$. The Italian domination number of a digraph $D$, denoted by $\gamma_{I}(D)$, is the minimum taken over the weights of all Italian dominating functions on $D$. A $\gamma_{I}(D)$-function is an Italian dominating function on $D$ with weight $\gamma_{I}(D)$.

The Italian dominating functions in graphs and digraphs have been studied in [1-7]. The authors of [2] introduce the concept of Italian domination and give bounds, relating the Italian domination number to some other domination parameters. The authors of [3] characterize the trees $T$ with $\gamma(T)+1=\gamma_{I}(T)$ and also characterize the trees $T$ with $\gamma_{I}(T)=2 \gamma(T)$. After that, there are some studies on the cartesian products of undirected cycles or undirected paths in [4,8-10]. Recently, 
the author of [6] initiated the study of the Italian domination number in digraphs. In this article, we investigate the Italian domination numbers of $C_{m} \square C_{n}$ and $C_{m} \otimes C_{n}$.

The following results are useful to our study.

Proposition 1 ([6]). Let $D$ be a digraph of order $n$. Then $\gamma_{I}(D) \geq\left\lceil\frac{2 n}{2+\Delta^{+}(D)}\right\rceil$.

Proposition 2 ([6]). Let $D$ be a digraph of order $n$. Then $\gamma_{I}(D) \leq n$, and $\gamma_{I}(D)=n$ if and only if $\Delta^{+}(D), \Delta^{-}(D) \leq 1$.

Proposition 3 ([6]). If $D$ is a directed path or a directed cycle of order $n$, then $\gamma_{I}(D)=n$.

\section{The Italian Domination Numbers of Some Products of Directed Cycles}

In this section, we determine the exact values of $\gamma_{I}\left(C_{m} \square C_{n}\right)$ and $\gamma_{I}\left(C_{m} \otimes C_{n}\right)$.

First, we consider $C_{m} \square C_{n}$. We denote the vertex set of a directed cycle $C_{m}$ by $\{1,2, \ldots, m\}$, and assume that $i \rightarrow i+1$ is an arc of $C_{m}$. For every vertex $(i, j) \in V\left(C_{m} \square C_{n}\right)$, the first and second components are considered modulo $m$ and $n$, respectively. For each $1 \leq k \leq n$, we denote by $C_{m}^{k}$ the subdigraph of $C_{m} \square C_{n}$ induced by the set $\{(j, k) \mid 1 \leq j \leq m\}$. Note that $C_{m}^{k}$ is isomorphic to $C_{m}$. Let $f$ be a $\gamma_{I}\left(C_{m} \square C_{n}\right)$-function and set $a_{k}=\sum_{x \in V\left(C_{m}^{k}\right)} f(x)$. Then $\gamma_{I}\left(C_{m} \square C_{n}\right)=\sum_{k=1}^{n} a_{k}$. It is easy to see that $C_{m} \square C_{n}$ is isomorphic to $C_{n} \square C_{m}$. So, $\gamma_{I}\left(C_{m} \square C_{n}\right)=\gamma_{I}\left(C_{n} \square C_{m}\right)$.

Theorem 1. If $m=2 r$ and $n=2 s$ for some positive integers $r, s$, then $\gamma_{I}\left(C_{m} \square C_{n}\right)=\frac{m n}{2}$.

Proof. Define $f: V\left(C_{m} \square C_{n}\right) \rightarrow\{0,1,2\}$ by

$$
f((2 i-1,2 j-1))=f((2 i, 2 j))=1
$$

for each $1 \leq i \leq r$ and $1 \leq j \leq s$, and

$$
f((x, y))=0
$$

otherwise. It is easy to see that $f$ is an IDF of $C_{m} \square C_{n}$ with weight $\frac{m n}{2}$ and so $\gamma_{I}\left(C_{m} \square C_{n}\right) \leq \frac{m n}{2}$. Since $\Delta^{+}(D)=2$, it follows from Proposition 1 that $\gamma_{I}\left(C_{m} \square C_{n}\right) \geq \frac{m n}{2}$. Thus, we have $\gamma_{I}\left(C_{m} \square C_{n}\right)=$ $\frac{m n}{2}$.

Theorem 2. For an odd integer $n \geq 3, \gamma_{I}\left(C_{2} \square C_{n}\right)=n+1$.

Proof. Define $f: V\left(C_{2} \square C_{n}\right) \rightarrow\{0,1,2\}$ by

$$
f((1,2 j-1))=1
$$

for each $1 \leq j \leq \frac{n+1}{2}$

$$
f((2,2 j))=1
$$

for each $1 \leq j \leq \frac{n-1}{2}$

$$
f((2, n))=1
$$

and

$$
f((x, y))=0
$$

otherwise. It is easy to see that $f$ is an IDF of $C_{2} \square C_{n}$ with weight $n+1$ and so $\gamma_{I}\left(C_{2} \square C_{n}\right) \leq n+1$.

Now we claim that $\gamma_{I}\left(C_{2} \square C_{n}\right) \geq n+1$. Suppose to the contrary that $\gamma_{I}\left(C_{2} \square C_{n}\right) \leq n$. Let $f$ be a $\gamma_{I}\left(C_{2} \square C_{n}\right)$-function. If $a_{k}=0$ for some $k$, assume without loss of generality $k=3$, then $f((1,3))=$ 
$f((2,3))=0$. To dominate the vertices $(1,3)$ and $(2,3)$, we must have $f((1,2))=f((2,2))=2$. Define $g: V\left(C_{2} \square C_{n}\right) \rightarrow\{0,1,2\}$ by

$$
g((1,2))=g((2,1))=g((2,3))=1, g((2,2))=0
$$

and

$$
g((x, y))=f((x, y))
$$

otherwise. Then $g$ is an IDF of $C_{2} \square C_{n}$ with a weight less than $\omega(f)$, which is a contradiction. Thus, $a_{k} \geq 1$ for each $k$. By assumption, $a_{k}=1$ for each $k$. Without loss of generality, we assume that $f((1,2))=1$. To dominate $(2,2)$, we must have $f((2,1))=1$. Since $a_{3}=1$ and $f((2,2))=0$, we have $f((2,3))=1$. By repeating this process, we obtain $f((1,2 i))=1$ for each $1 \leq i \leq \frac{n-1}{2}$, $f((2,2 i-1))=1$ for $1 \leq i \leq \frac{n+1}{2}$ and $f((x, y))=0$ otherwise. However, the vertex $(1,1)$ is not dominated, and this is a contradiction. Thus we have $\gamma_{I}\left(C_{2} \square C_{n}\right) \geq n+1$. This completes the proof.

Theorem 3. For an integer $n \geq 3, \gamma_{I}\left(C_{3} \square C_{n}\right)=2 n$.

Proof. When $n=3 r$ for some positive integer $r$, define $f_{0}: V\left(C_{3} \square C_{n}\right) \rightarrow\{0,1,2\}$ by

$$
f_{0}((1,3 j+1))=f_{0}((2,3 j+1))=1
$$

for each $0 \leq j \leq n-1$,

$$
f_{0}((2,3 j+2))=f_{0}((3,3 j+2))=1
$$

for each $0 \leq j \leq n-1$,

$$
f_{0}((1,3 j+3))=f_{0}((3,3 j+3))=1
$$

for each $0 \leq j \leq n-1$ and

$$
f_{0}((x, y))=0
$$

otherwise.

When $n=3 r+1$ for some positive integer $r$, define $f_{1}: V\left(C_{3} \square C_{n}\right) \rightarrow\{0,1,2\}$ by

$$
f_{1}((2, n))=f_{1}((3, n))=1
$$

and

$$
f_{1}((x, y))=f_{0}((x, y))
$$

otherwise.

When $n=3 r+2$ for some positive integer $r$, define $f_{2}: V\left(C_{3} \square C_{n}\right) \rightarrow\{0,1,2\}$ by

$$
f_{2}((1, n-1))=f_{2}((2, n-1))=f_{2}((1, n))=f_{2}((3, n))=1
$$

and

$$
f_{2}((x, y))=f_{0}((x, y))
$$

otherwise. It is easy to see that $f_{i}(i=0,1,2)$ is an IDF of $C_{3} \square C_{n}$ with weight $2 n$ and so $\gamma_{I}\left(C_{3} \square C_{n}\right) \leq 2 n$.

Now we prove that $\gamma_{I}\left(C_{3} \square C_{n}\right) \geq 2 n$. Let $f$ be a $\gamma_{I}\left(C_{3} \square C_{n}\right)$-function.

Claim 1. $a_{k} \geq 1$ for each $1 \leq k \leq n$. 
Suppose to the contrary that $a_{k}=0$ for some $k$, say $k=n$. To dominate $(1, n),(2, n)$ and $(3, n)$, we must have $f((1, n-1))=f((2, n-1))=f((3, n-1))=2$. However, the function $g$ defined by

$$
\begin{gathered}
g((1, n-1))=g((2, n-1))=g((3, n-1))=1, \\
g((1, n))=g((2, n))=1
\end{gathered}
$$

and

$$
g((x, y))=f((x, y))
$$

otherwise, is an IDF of $C_{3} \square C_{n}$ with a weight less than $\omega(f)$. This is a contradiction.

We choose a $\gamma_{I}\left(C_{3} \square C_{n}\right)$-function $h$ so that the size of $M_{h}:=\left\{k \mid a_{k}=1\right\}$ is as small as possible.

Claim 2. $\left|M_{h}\right|=0$.

Suppose to the contrary that $\left|M_{h}\right| \geq 1$. Without loss of generality, assume that $a_{n}=1$ and $h((1, n))=1$. To dominate $(2, n)$ and $(3, n)$, we must have $h((2, n-1))=1$ and $h((3, n-1))=2$. If $n=3$, then clearly $a_{1} \geq 2$ and so $\gamma_{I}\left(C_{3} \square C_{3}\right) \geq 6$. However, when $n=3$, the previously defined function $f_{0}$ is a $\gamma_{I}\left(C_{3} \square C_{3}\right)$-function such that $\omega\left(f_{0}\right)=6$ and $\left|M_{f_{0}}\right|=0$. This contradicts the choice of $h$. From now on, assume $n \geq 4$. We divide our consideration into the following two cases.

Case 1. $a_{n-2}=1$.

By the same argument as above, we have $a_{n-3} \geq 3$. So $a_{n-3}+a_{n-2}+a_{n-1}+a_{n} \geq 8$. If $n=4$, then the previously defined function $f_{1}$ induces a contradiction. Suppose $n \geq 5$. Since $a_{n-4} \geq 1$ by Claim $1, h((i, n-4))=1$ or 2 for some $i \in\{1,2,3\}$. Without loss of generality, we may assume $h((1, n-4))=1$ or 2 . Define $t: V\left(C_{3} \square C_{n}\right) \rightarrow\{0,1,2\}$ by

$$
\begin{aligned}
& t((1, n-3))=t((2, n-2))=t((1, n-1))=t((2, n))=0, \\
& t((2, n-3))=t((1, n-2))=t((2, n-1))=t((1, n))=1, \\
& t((3, n-3))=t((3, n-2))=t((3, n-1))=t((3, n))=1
\end{aligned}
$$

and

$$
t((x, y))=h((x, y))
$$

otherwise. Then it is easy to see that $t$ is an IDF of $C_{3} \square C_{n}$ such that $\left|M_{t}\right|<\left|M_{h}\right|$. This contradicts the choice of $h$.

Case 2. $a_{n-2} \geq 2$.

Now $a_{n-2}+a_{n-1}+a_{n} \geq 6$. Since $a_{n-3} \geq 1$ by Claim $1, h((i, n-3))=1$ or 2 for some $i \in\{1,2,3\}$. Without a loss of generality, we may assume $h((1, n-3))=1$ or 2 . Define $t: V\left(C_{3} \square C_{n}\right) \rightarrow\{0,1,2\}$ by

$$
\begin{aligned}
& t((1, n-2))=t((2, n-1))=t((3, n))=0, \\
& t((2, n-2))=t((1, n-1))=t((1, n))=1, \\
& t((3, n-2))=t((3, n-1))=t((2, n))=1
\end{aligned}
$$

and

$$
t((x, y))=h((x, y))
$$

otherwise. Then it is easy to see that $t$ is an IDF of $C_{3} \square C_{n}$ such that $\left|M_{t}\right|<\left|M_{h}\right|$. This contradicts the choice of $h$.

By Claims 1 and 2, we have $\gamma_{I}\left(C_{3} \square C_{n}\right) \geq 2 n$. This completes the proof. 
Next, we consider $C_{m} \otimes C_{n}$. We denote the vertex set of a directed cycle $C_{m}$ by $\{1,2, \ldots, m\}$, and assume that $i \rightarrow i+1$ is an arc of $C_{m}$. For every vertex $(i, j) \in V\left(C_{m} \otimes C_{n}\right)$, the first and second components are considered modulo $m$ and $n$, respectively. For each $1 \leq k \leq n$, we denote by $C_{m}^{k}$ the subdigraph of $C_{m} \otimes C_{n}$ induced by the set $\{(j, k) \mid 1 \leq j \leq m\}$. Note that $C_{m}^{k}$ is isomorphic to $C_{m}$. Let $f$ be a $\gamma_{I}\left(C_{m} \otimes C_{n}\right)$-function and set $a_{k}=\sum_{x \in V\left(C_{m}^{k}\right)} f(x)$. Then $\gamma_{I}\left(C_{m} \otimes C_{n}\right)=\sum_{k=1}^{n} a_{k}$.

Lemma 1. For positive integers $m, n \geq 2, \gamma_{I}\left(C_{m} \otimes C_{n}\right) \geq\left\lceil\frac{m n}{2}\right\rceil$.

Proof. Note that the vertices of $C_{m}^{k}$ are dominated by vertices of $C_{m}^{k-1}$ or $C_{m}^{k}$. It suffices to verify that $\sum_{k=1}^{n} a_{k} \geq\left\lceil\frac{m n}{2}\right\rceil$. In order to do so, we claim $a_{k}+a_{k+1} \geq m$ for each $k$. First of all, we assume that $a_{k+1}=0$. Then, to dominate $(i, k+1)$ for each $1 \leq i \leq m$, we must have

$$
f((i-1, k))+f((i, k)) \geq 2
$$

Then, $2 a_{k}=\sum_{i=1}^{m}(f((i-1, k))+f((i, k))) \geq 2 m$ and hence $a_{k}+a_{k+1} \geq m$. If $a_{k+1}=t>0$, then there will be at least $m-t$ vertices in $V_{0}$ that will be dominated only by vertices of $C_{m}^{k}$. This fact induces $a_{k} \geq m-t$ and so $a_{k}+a_{k+1} \geq m$. Therefore, we have

$$
2 \gamma_{I}\left(C_{m} \otimes C_{n}\right)=2 \sum_{k=1}^{n} a_{k}=\sum_{k=1}^{n}\left(a_{k}+a_{k+1}\right) \geq n m .
$$

This completes the proof.

Theorem 4. For positive integers $m, n \geq 2, \gamma_{I}\left(C_{m} \otimes C_{n}\right)=\left\lceil\frac{m n}{2}\right\rceil$.

Proof. We divide our consideration into the following two cases.

Case 1. $m$ or $n$ is even.

Since $C_{m} \otimes C_{n}$ is isomorphic to $C_{n} \otimes C_{m}$, we may assume that $n=2 s$ for some positive integers $s$. Define $f: V\left(C_{m} \otimes C_{n}\right) \rightarrow\{0,1,2\}$ by

$$
f((i, 2 j-1))=1
$$

for each $1 \leq i \leq m$ and $1 \leq j \leq s$, and

$$
f((x, y))=0
$$

otherwise. It is easy to see that $f$ is an IDF of $C_{m} \otimes C_{n}$ with weight $\frac{m n}{2}$ and so $\gamma_{I}\left(C_{m} \otimes C_{n}\right) \leq\left\lceil\frac{m n}{2}\right\rceil$. Thus, it follows from Lemma 1 that $\gamma_{I}\left(C_{m} \otimes C_{n}\right)=\left\lceil\frac{m n}{2}\right\rceil$.

Case 2. $m=2 r+1, n=2 s+1$ for some positive integers $r, s$.

Define $f: V\left(C_{m} \otimes C_{n}\right) \rightarrow\{0,1,2\}$ by

$$
f((2 i+1,2 j+1))=1
$$

for each $0 \leq i \leq r$ and $0 \leq j \leq s$,

$$
f((2 i, 2 j))=1
$$

for each $1 \leq i \leq r$ and $1 \leq j \leq s$ and

$$
f((x, y))=0
$$

otherwise. It is easy to see that $f$ is an IDF of $C_{m} \otimes C_{n}$ with weight $(r+1)(s+1)+r s$ and so $\gamma_{I}\left(C_{m} \otimes C_{n}\right) \leq\left\lceil\frac{m n}{2}\right\rceil$. Thus, it follows from Lemma 1 that $\gamma_{I}\left(C_{m} \otimes C_{n}\right)=\left\lceil\frac{m n}{2}\right\rceil$. 


\section{Conclusions}

In this article, we determined the exact values of $\gamma_{I}\left(C_{2} \square C_{l}\right), \gamma_{I}\left(C_{3} \square C_{l}\right)$ and $\gamma_{I}\left(C_{m} \square C_{n}\right)$ for an integer $l$ and even integers $m, n$. The other cases are still open. We conclude by giving a conjecture.

Conjecture 1. For an odd integer $n, \gamma_{I}\left(C_{4} \square C_{n}\right)=2 n+2$.

Acknowledgments: The author would like to thank anonymous referees for their valuable comments.

Funding: This research was supported by the Basic Science Research Program through the National Research Foundation of Korea funded by the Ministry of Education (2020R111A1A01055403).

Conflicts of Interest: The author declares no conflict of interest.

\section{References}

1. Fan, W.; Ye, A.; Miao, F.; Shao, Z.; Samodivkin, V.; Sheikholeslami, S.M. Outer-Independent Italian Domination in Graphs. IEEE Access 2019, 7, 22756-22762. [CrossRef]

2. Chellali, M.; Haynes, T.W.; Hedetniemi, S.T.; McRae, A.A. Roman \{2\}-domination. Discret. Appl. Math. 2016, 204, 22-28. [CrossRef]

3. Henning, M.A.; Klostermeyer, W.F. Italian domination in trees. Discret. Appl. Math. 2017, 217, 557-564. [CrossRef]

4. Gao, H.; Wang, P.; Liu, E.; Yang, Y. More results on Italian domination in $C_{n} \square C_{m}$. Mathematics 2020, 8, 465. [CrossRef]

5. Gao, H.; Xi, C.; Li, K.; Zhang, Q.; Yang, Y. The Italian domination numbers of generalized Petersen graphs $P(n, 3)$. Mathematics 2019, 7, 714. [CrossRef]

6. Volkmann, L. Italian domination in digraphs. J. Combin. Math. Combin. Comput. to appear.

7. Volkmann, L. The Italian domatic number of a digraph. Commun. Comb. Optim. 2019, 4, 61-70.

8. Stepien, Z.; Szymaszkiewicz, A.; Szymaszkiewicz, L.; Zwierzchowski, M. 2-Rainbow domination number of $C_{n} \square C_{5}$. Discret. Appl. Math. 2014, 107, 113-116. [CrossRef]

9. Gao, H.; Xu, T.T.; Yang, Y.S. Bagging approach for Italian domination in $C_{n} \square P_{m}$. IEEE Access 2019, 7, 105224-105234. [CrossRef]

10. Li, Z.P.; Shao, Z.H.; Xu, J. Weak $\{2\}$-domination number of Cartesian products of cycles. J. Comb. Optim. 2018, 35, 75-85. [CrossRef]

(C) 2020 by the author. Licensee MDPI, Basel, Switzerland. This article is an open access article distributed under the terms and conditions of the Creative Commons Attribution (CC BY) license (http:// creativecommons.org/licenses/by/4.0/). 